\title{
Manufactured by software: SDN-enabled multi- operator composite services with the 5G Exchange
}

\author{
Gergely Biczók (MTA-BME Future Internet Research Group, biczok@tmit.bme.hu) \\ Manos Dramitinos (Athens Univ. of Economics and Business, mdramit@aueb.gr) \\ Laszlo Toka (MTA-BME Information Systems Research Group, toka@tmit.bme.hu) \\ Poul E. Heegaard (Norwegian Univ. of Science and Technology, \\ poul.heegaard@item.ntnu.no) \\ Håkon Lønsethagen (Telenor, hakon.lonsethagen@telenor.com)
}

\section{Abstract}

Foreseen $5 \mathrm{G}$ verticals hold the promise of being true value-added services, hence bringing significant income to their respective providers. However, the nature of these verticals are very demanding in terms of both economic and technical requirements, such as multi-operator cooperation, end-to-end quality assurance and the unified orchestration of network and cloud resources. Existing systems fall short of satisfying these requirements, but emerging network softwarization and resource virtualization technologies, such as Software-Defined Networking (SDN) and Network Function Virtualization (NFV) show promise for being key enablers in such context. In this paper, we introduce the 5G Exchange (5GEx) concept that builds on SDN and $\mathrm{NFV}$, and facilitates the provisioning of multi-operator $5 \mathrm{G}$ services by means of inter-operator management and orchestration of virtualized network, compute and storage resources. We present potential 5GEx use cases, conceptual architecture and value proposition. We also outline open research questions on how to exchange information in such a co-opetitive environment, and provide an outlook on the impact of 5GEx on a network service provider's business and operation.

\section{Introduction}

Internet services have evolved rapidly, covering all aspects of communication and infotainment. Services such as video-on-demand and online gaming are already popular, while additional verticals, also integrating cloud and the Internet of Things (IOT), are envisioned in the context of 5G [1]. Efficient provisioning of these services as high-value products in the market requires service-aware routing, end-to-end Quality of Service (QoS) assurance including dependability aspects, elastic resource and dynamic service orchestration (over network and cloud infrastructures) and flexible service management. These requirements are currently not met by Best Effort Internet and the inherent shortcomings of a single-traffic-class approach: Large buffers for statistical multiplexing gain inevitably increase delay; there is no way to protect critical over non-critical traffic; the flow control protocols cannot efficiently adapt to congestion and match application requirements with network capabilities, while BGP does not allow for multiple choices for service-aware routing of delay-tolerant versus delay-critical traffic so as to both optimize QoS and load balance the network. 
Internet service layer stakeholders buy and sell Internet services and are categorized as Connectivity Providers, Information Providers, also referred to as Over-The-Top (OTTs), and end-users. Connectivity Providers, also referred to as Network Service Providers (NSPs), normally own their network and are responsible for the provisioning of its functionalities. The fact that multiple network (including $5 \mathrm{G}$ radio access), cloud and OTT stakeholders comprise the multi-actor value chain of $5 \mathrm{G}$ services, inevitably calls for multi-operator business and service coordination jointly over the network, compute and storage domain. Unfortunately, currently there is no open and global solution to multi-service internetworking, resulting in costly, legacy, provider-specific service provisioning. This limits the potential of standardized integration of network, compute and storage infrastructure under a unified service orchestration, control and management framework. Thus, it is insufficient to carry the $5 \mathrm{G}$ value creation at the edge of the networks across the backbones hindering the $5 \mathrm{G}$ services value creation. The softwarization of the network control plane and the virtualization of resources can be powerful enablers in the context of a novel exchange mechanism supporting ondemand service creation, standard resource abstractions, resource trading and flexible interprovider Service Level Agreements (SLAs). Such an exchange framework has the potential to remove the inherent shortcomings of today's solutions, enables $5 \mathrm{G}$ value creation at the network edge and matching of requirements of $5 \mathrm{G}$ applications and services to properties of connectivity services end-to-end over the virtualized network infrastructure.

Multi-operator services currently rely on best-effort connectivity enabled by service-agnostic interconnection agreements pertaining to inter-domain traffic aggregates. As a result, services experience unpredictable network performance that mostly depends on insufficient and inefficient overprovisioning [2]. Paired with the increasing overall traffic demand and the limited incentives for investing in new infrastructure, 5G services provisioning is a formidable challenge. Overprovisioning is a bad strategy for parts of the network, since a capacity upgrade also brings substantial benefit to a less well-provisioned but interconnected network, making the latter also more attractive to end-users [3]. Thus, though both networks could benefit from an upgrade, selfishly maintaining a low quality interconnection is often a dominant strategy for large ISPs [4].

In this paper, we introduce the novel 5G Exchange (5GEx) concept: drawing on the disruptive innovative technologies of softwarized service orchestration and control (SDN) and resource and network function virtualization (NFV), 5GEx is positioned to be a key market enabler for the provisioning of multi-operator infrastructure services and a catalyst for materializing the value of $5 \mathrm{G}$ verticals. From the technical resource orchestration aspect, 5GEx enables the transition from dedicated physical networks and resources for different applications to a "network factory", where resources and network functions are traded on-demand and new services are "manufactured by software" (see Figure 1). Orchestration of the heterogeneous resource domains are achieved via virtualization of resources and network functions and a smart slicing method operating over those virtualized entities. 


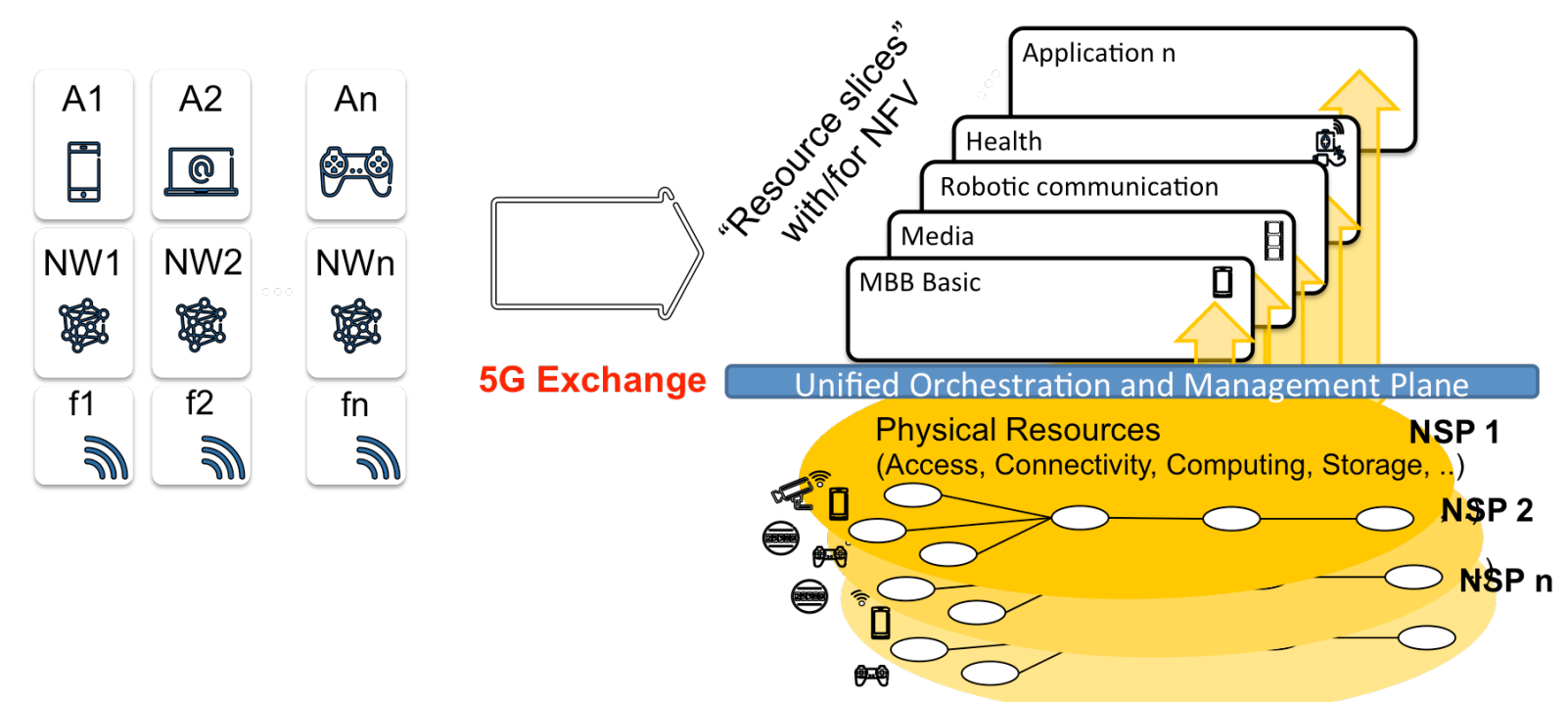

Figure 1 From dedicated physical resources to network factory: services manufactured by software

The rest of the paper is organized as follows. Section 2 presents the state-of-the-art in multioperator interconnection alternatives. Section 3 presents the benefits of SDN and NFV, and how these characteristics enable the proposed exchange framework. Section 4 introduces the conceptual design of the $5 \mathrm{G}$ Exchange. Section 5 discusses operational challenges and opportunities in the context of the exchange from the operator's aspect. Finally, Section 6 concludes the paper.

\section{Multi-operator interconnection: state-of-the-art}

Multi-operator services have been implicitly supported over the Internet by means of pure connectivity services and interconnection agreements between the operators. Networks rely on BGP to build the Internet connectivity graph. They solely exchange BGP announcements and data; interconnection agreements specify whether and how each network should accept and terminate or forward the traffic coming from a neighboring network. Existing peering and transit interconnection agreements do not provide any type of service-aware routing and management or QoS assurance, and pertain to inter-domain traffic aggregates of multiple services (elastic and inelastic).

From an economic standpoint, the work in [3] points out inefficiencies, such as unfair revenue distribution among providers which discourages network upgrades. The current "walled garden" digital service provisioning regime, i.e., operators focusing on intra-domain services for their customer base while being reluctant to cooperate with other operators beyond peering and transit, results in well-known business and economic inefficiencies. This motivates an increasing research and business interest in providing solutions for enabling sustainable ecosystems where services relying on open, agile, elastic management, as well as quality assurance can be efficiently provisioned [2].

The ETICS research project has attempted to mitigate these inefficiencies by complementing traditional interconnection peering and transit products with additional products for the 
provisioning of quality assurance to the inter-domain interconnection services termed Assured Quality (ASQ) products [5]. ASQ products support technology-agnostic paths of assured performance and attributes such as IP addresses/prefixes, delay, jitter, bandwidth; SLA attributes are propagated via an overlay of Path Communication Elements, horizontal and vertical interfaces that map them to and enforce them in the underlying networks. ASQ products allow a finer degree of traffic control over inter-domain network paths and regions, while peering pertains to two networks and transit offers global connectivity to the buyer.

Evolving from a separate industry strand, the IP Exchange (IPX) is a non-Internet telecommunications interconnection model developed by GSMA for the exchange of IP-based traffic between customers of separate mobile and fixed operators as well as ISPs; essentially, a privately managed IP backbone [6]. IPX, in contrast to the Internet approach, inherently supports QoS interconnection with respective SLAs and cascading payments between operators. There are two characteristics of IPX, which go against IPX becoming a truly global solution. First, IPX uses Network Address Translators and per session gating at IPX border routers making it hard to scale at a global level. Second, IPX is required to be compatible with legacy voice services, adding additional complexity. These characteristics render IPX too complex and expensive for serving as a general purpose backbone for $5 \mathrm{G}$ services.

From a cloud provider's standpoint, cloud federations and exchanges are gaining momentum, including data center interconnection over federated infrastructure. A typical example is OnApp [7], a federation of cloud providers with cloud and CDN product offerings of fine geospatial granularity worldwide. In addition, UK-based CloudStore [8] supports public, private and hybrid clouds and laaS, SaaS, PaaS and Specialist Cloud Services. While certainly a hotbed of technical and business innovation recently, solutions from the cloud domain have to be complemented and harmonized with end-to-end assured quality connectivity to successfully provision $5 \mathrm{G}$ services.

\section{Enabling technologies: SDN, NFV and SFC}

While there has been tremendous activity in the networking research community with regard to service quality assurance spanning multiple decades, we believe that the time has just become ripe for capitalizing on a well-designed solution. Two of the key requirements have just materialized: 1 ) we have the "killer apps" in form of value-added $5 G$ verticals whose value chain is inherently multi-operator in general and 2) enabling technology has just reached the needed maturity level, in the form of Software-Defined Networking (SDN), Network Function Virtualization (NFV) and Service Function Chaining (SFC), and how these technologies can be integrated around the concept of network (and later compute and storage resource) slices.

SDN, in its original interpretation, decouples control from the data plane (and therefore vendorspecific hardware), assigning it to a software controller. SDN simplifies routers and switches and can improve data throughput and reduce congestion via traffic management and optimized resource allocation applied by the controller. In the context of 5G, SDN enables service-aware routing, flow-level quality assurance and efficient dynamic resource management by a logically centralized control logic. The defining benefit of SDN for us, however, lies in its ability to provide an abstraction of the physical network infrastructure. SDN 
provides network programmability: several customized network slices can be configured in parallel using the same physical and logical infrastructure. Thus, one physical network can support a variety of services in an optimal way.

NFV allows for a network function to be implemented in software instead of by a piece of dedicated hardware. This concept comes with inherent scalability supporting the delivery of on-demand, dynamically re-scalable and global services. For us, the key feature of NFV lies in its ability to execute NFs independently of location; this essentially means that the same network function can be executed at different locations for different network slices. Hence, a service-aware virtual network environment is created by the actual placement of NFs.

SFC is not a novel concept in itself: the delivery of end-to-end services often requires various network service (e.g., firewall) and application-specific functions (e.g., HTTP header processing) to be "chained". However, if functions are virtualized and can be placed at arbitrary physical location, and SDN policies are used to steer data traffic through them in a servicespecific manner, we have the ultimate elastic service environment: instantly, rapid creation, destruction, scaling and migration of service functions and (with an agile service insertion model) services become possible.

SDN and NFV allow architects to build systems with greater degree of freedom and abstractions, thus network flexibility: with their help the vertical networking of yesterday can be broken down to building blocks that can be chained together to suit the services to be supported. We refer to this concept as service-aware slicing; we believe it has the potential to enable the highly-coveted flexibility in service provisioning and delivery (the "Holy Grail of 5G"), while reducing overall costs at the same time.

SDN is also a key enabler from a multi-operator collaboration perspective, as it allows for the direct expression of flexible policies potentially tailored to different applications and service quality requirements (a potential stepping stone for an improved SLA framework). Drawing from (a simplified version of) this idea, the project SDX (SDN eXchange point) [9] proposes to deploy SDN-capable switches at Internet Exchange Points (IXPs) in order to make a step from conventional hop-by-hop, destination-based forwarding and enable participating ISPs to apply diverse actions on packets from the IXP such as inbound traffic engineering, redirection of traffic to middleboxes or load balancing. We believe that SDX is a step in the right direction, and serves as an important precursor to 5GEx.

\section{The 5G Exchange concept}

The 5G Exchange project aims at enabling $5 G$ verticals by designing an exchange framework capable of handling the orchestration of both network and cloud resources over multiple technological and administrative domains [10]. Apart from catering to the needs of future $5 \mathrm{G}$ services, 5GEx also has an objective of overcoming the historical technological and market fragmentation of the European telecommunications sector by bootstrapping operator collaboration with regard to infrastructure services. Such infrastructure services (and associated resources) provide the foundation of all $5 \mathrm{G}$ verticals making use of cloud and networking services, apart from the radio interface itself. The envisioned $5 \mathrm{G}$ Exchange will 
enable operators to buy, sell and integrate virtual resources and services, thus enabling onestop shopping for their customers: it suffices for the customer to contact and contract with a single operator, who will then outsource part of its commitments to other operators given the lack of geographical footprint or available resources. Furthermore, the generic, open and standardized offering of various connectivity modes supported with other $5 \mathrm{G}$ capabilities, will enable the numerous SMEs and content providers to differentiate and monetize their online content and application offerings. This will open up new venues of innovation for many businesses and verticals yet unseen, in various consumer, business and public sector markets.

$5 G$ services extend personal communication and video services with the integration of Cloud, IoT and machine-to-machine communication into the $5 \mathrm{G}$ architecture and service model. NGMN [1] specifies 24 use cases for 5G, to be delivered across various devices (smartphone, wearable, machine module) grouped to 8 families, along with related customer-facing services (verticals): 3 families of high-speed broadband access everywhere with HD video sharing as vertical, massive loT with sensor/smart home networks as verticals, 3 families of lifeline/ultrareliable communications with e-health/telemedicine as vertical and broadcast services for infotainment. These use case families and verticals motivate the wholesale infrastructure services needed to support them, enabled by resource virtualization, network softwarization and service orchestration and management of the proposed 5GEx multi-operator exchange. $5 \mathrm{GEx}$ can be seen as the $5 \mathrm{G}$ evolution of exchange environments such as IPX, SDX and IXPs; a core subset of $5 \mathrm{G}$ infrastructure services envisioned that are capable of supporting the aforementioned use cases and verticals are Connectivity (e.g., VPN+), Virtual Network Function as a Service (VNFaaS, e.g., vCDN) and Anything-as-a-Service (XaaS, e.g., Gi-LAN).

Connectivity is a use case family of wholesale connectivity services over multiple domains, capable of supporting next-generation connectivity verticals such as VPN+. VPN+ denotes an improved Virtual Private Network service (aimed at an enterprise customer) with a Networkas-a-Service (NaaS) element such as partial topology description and dependability requirements. The vCDN use case implements a virtual Content Distribution Network, where a video portal (customer) purchases the right to use the CDN facilities of a CDN provider: this also involve storage resources. XaaS represents the most challenging use case in that it potentially involves the full range of network, compute and storage resources with strict performance and dependability guarantees, e.g., ultra-low latency and adequate computational capacity, to enable demanding verticals such as industrial robotics and Mobile Edge Computing (MEC) scenarios. A very concrete instantiation of XaaS inspired by a true operator need is international mobile data roaming (referred to as the Gi-LAN use case). Since the EU will ban mobile roaming fees within Europe, a drastic increase of roaming data usage is expected. The normal process of roaming would involve building tunnels back to the home Packet GateWay (h-PGW) through international exchange points: an expensive and unfavorably scaling mechanism. Moving the h-PGW and the entire Gi-LAN functionality to the roaming operator's data center results in a cheaper and dynamically scalable solution. (While this list of use cases is clearly not exhaustive, they demonstrate the different expected capabilities of $5 \mathrm{G}$ Exchange well.)

The aforementioned wholesale 5GEx infrastructure services can efficiently serve verticals by relying on lower-level 5GEx fundamental services and SDN/NFV techniques, with the core element being the slice. A slice is defined as a managed set of $5 \mathrm{G}$ resources and network 
functions set up within the $5 \mathrm{G}$ system that is tailored to support a particular type of user or service. Note how the slice concept is an evolution of network slices as used in SDN also incorporating cloud resources and NFs. These slices are then instantiated on-demand using APIs exposed by the management plane, which provides dynamic orchestration for multilayer and multi-domain networks. SDN and NFV greatly simplify slice and service orchestration and management, as opposed to the traditional inter-working of legacy networks and clouds. 5GEx uses a) standard interface (1) for multi-domain orchestrator to translate the 5GEx customer service request to a chain of VNFs and underlying network, storage and cloud resource requirements b) standard interface (2) and respective SLAs for trading slices and 5GEx higherlevel services among 5GEx-enabled orchestrators and c) standard interface (3) for the management of own or leased - via interface (2) - resources. For a simplified conceptual architecture of 5GEx please refer to Figure 2. Precursor projects containing ideas and code for interfaces include ETICS (interface 2, [5]), UNIFY (interface 3, [11]) and T-NOVA (interface $1,[12])$.

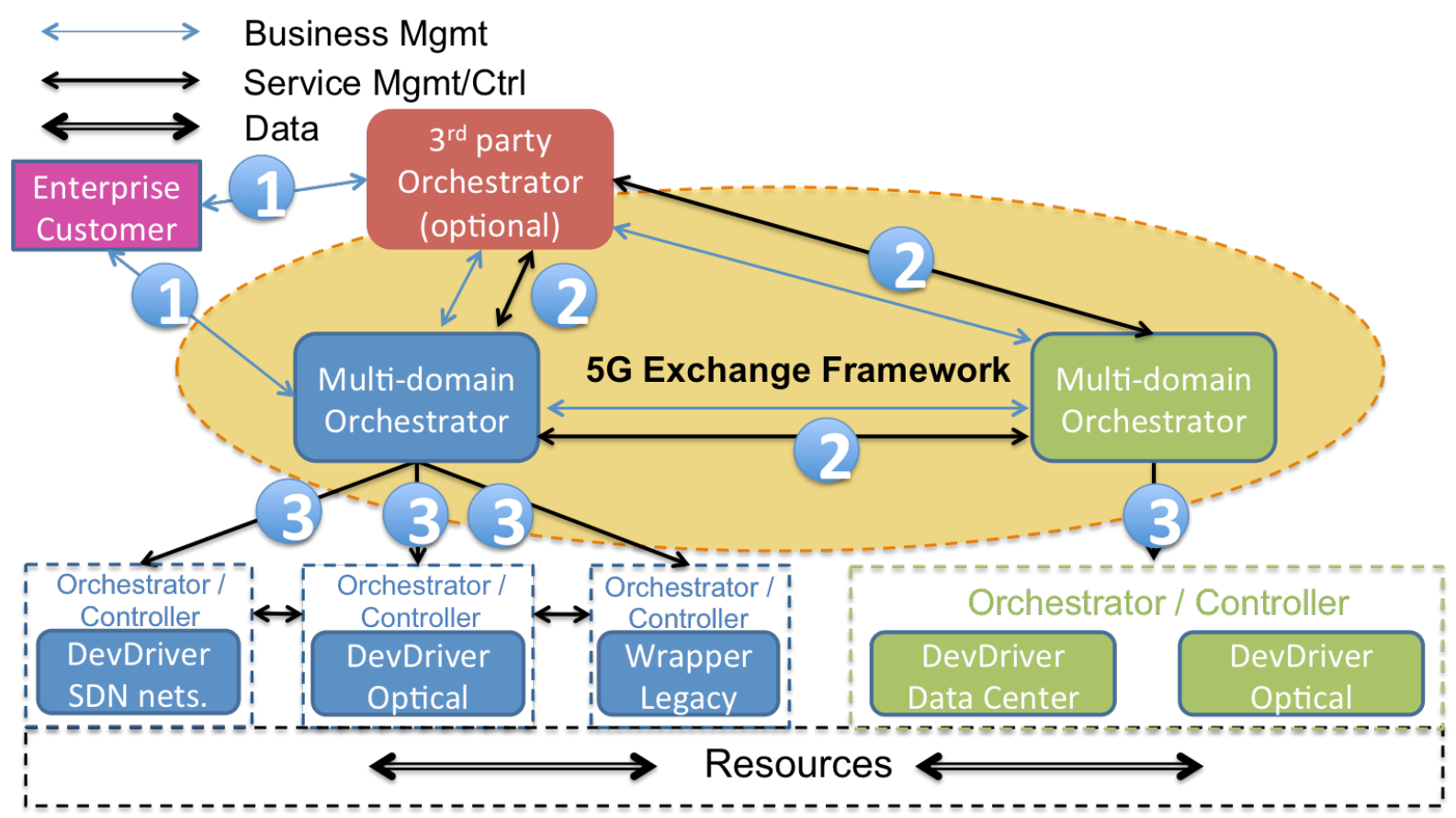

Figure 2 Simplified conceptual architecture of the 5G Exchange

The proposed $5 \mathrm{G}$ Exchange Framework supports a variety of specific deployments and coordination/collaboration models such as: i) "direct peering" at an already established local or remote IXP, ii) distributed multi-party collaboration, where the operators host the exchange mechanism in a distributed manner inside their own infrastructure, and iii) a dedicated (for-profit) Exchange Point Provider as a standalone entity, offering exchange point services. In addition, the 5G Exchange Framework also supports higher level abstractions and advanced models covering views, resources and services across several exchange points or PoPs. Also note that the customer-facing "3rd party Orchestrator" in Figure 2 is an optional role in the ecosystem, essentially referring to a virtual network operator who implements the multi-domain orchestrator functionality, but does not own an infrastructure.

The clear separation of functionality allows 5GEx to make the most out of SDN and NFV, creating an open agile management and orchestration environment where multi-operator services become only marginally more complex than single-operator services due to the 
common interfaces and functionality for the management of both own and leased services. There is an analogy here with the cloud ecosystem in terms of architecture and value proposition, with the various 5GEx layers [13] mapped to cloud resources and services ranging, e.g., from Amazon's S3 through EC2 to CloudFront high-level service, as depicted in Figure 3. Lower-level resources are the low-margin commodity building blocks of differentiated higher-level services for serving $5 \mathrm{G}$ verticals. At all levels of the value chain, we use the service concept appropriate for the given level. Virtual resources and NFs are composed into slices utilizing the Network Function Infrastructure as a Service (NFVlaaS) paradigm; slices make up infrastructure services, by the concept of Slice-as-a-Service (SlaaS); finally, infrastructure services comprise a custom $5 \mathrm{G}$ vertical, which in turn can be purchased by a customer residing outside the 5G Exchange (high profit margin). Therefore, intra-5GEx services by-design consider the needs of $5 G$ customer-facing services. The (potentially recursive) trading of slices and 5GEx services over interface (2) can support multiple coordination models, including push and pull, if goods are built on-demand or pre-built and part of a catalog, as well as distributed or centralized, whether the multi-domain orchestration is a centralized third-party service or implemented in a distributed fashion over multiple instances, one per 5GEx infrastructure service provider.
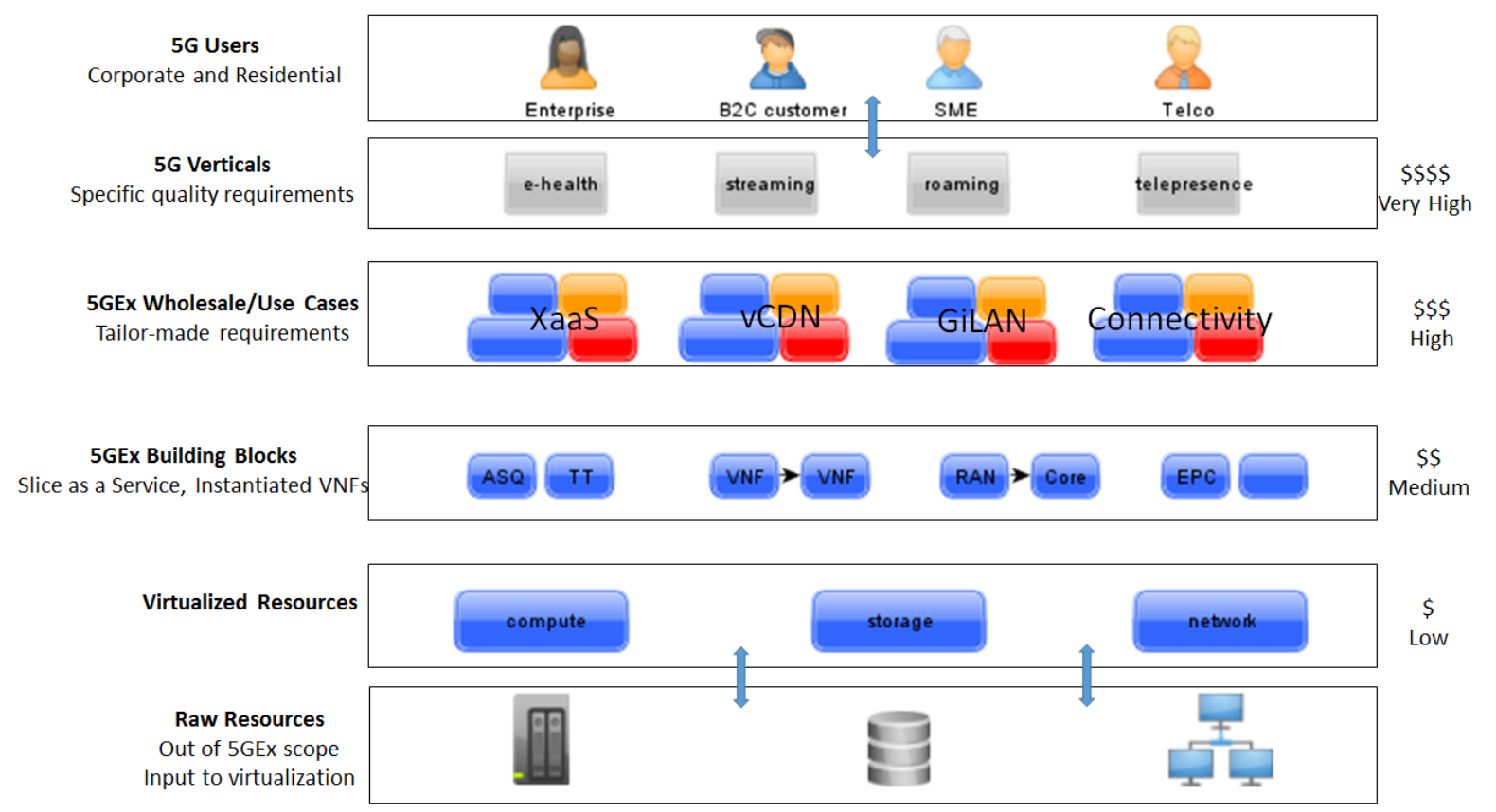

Figure 3 Levels of 5GEx goods and value proposition mapped to the current cloud ecosystem

Although the conceptual architecture and value proposition are clear, there are still some design challenges ahead, the most glaring focused on the exchange of information among 5GEx operators (see Figure 4 for an example with User 1 as customer and Op1 as customerfacing operator). On the one hand, SDN supports a rich set of possibilities for exchanging information as described in Section 3. On the other hand, what kind of information should be shared, with what granularity and how to calculate relevant KPIs and design corresponding SLAs are all mostly open questions. Naturally, there is a tradeoff between the business interests (sharing the least amount of information possible) and optimal end-to-end resource management guaranteeing assured quality (sharing the most precise information possible). Furthermore, virtualization involves aggregation of information about the physical resources, network topology and policies; multiple levels of virtualization in 5GEx makes matters even 
more complex. Thus, the chosen method of information exchange has far reaching consequences with regard to both performance and dependability of the provisioned services.

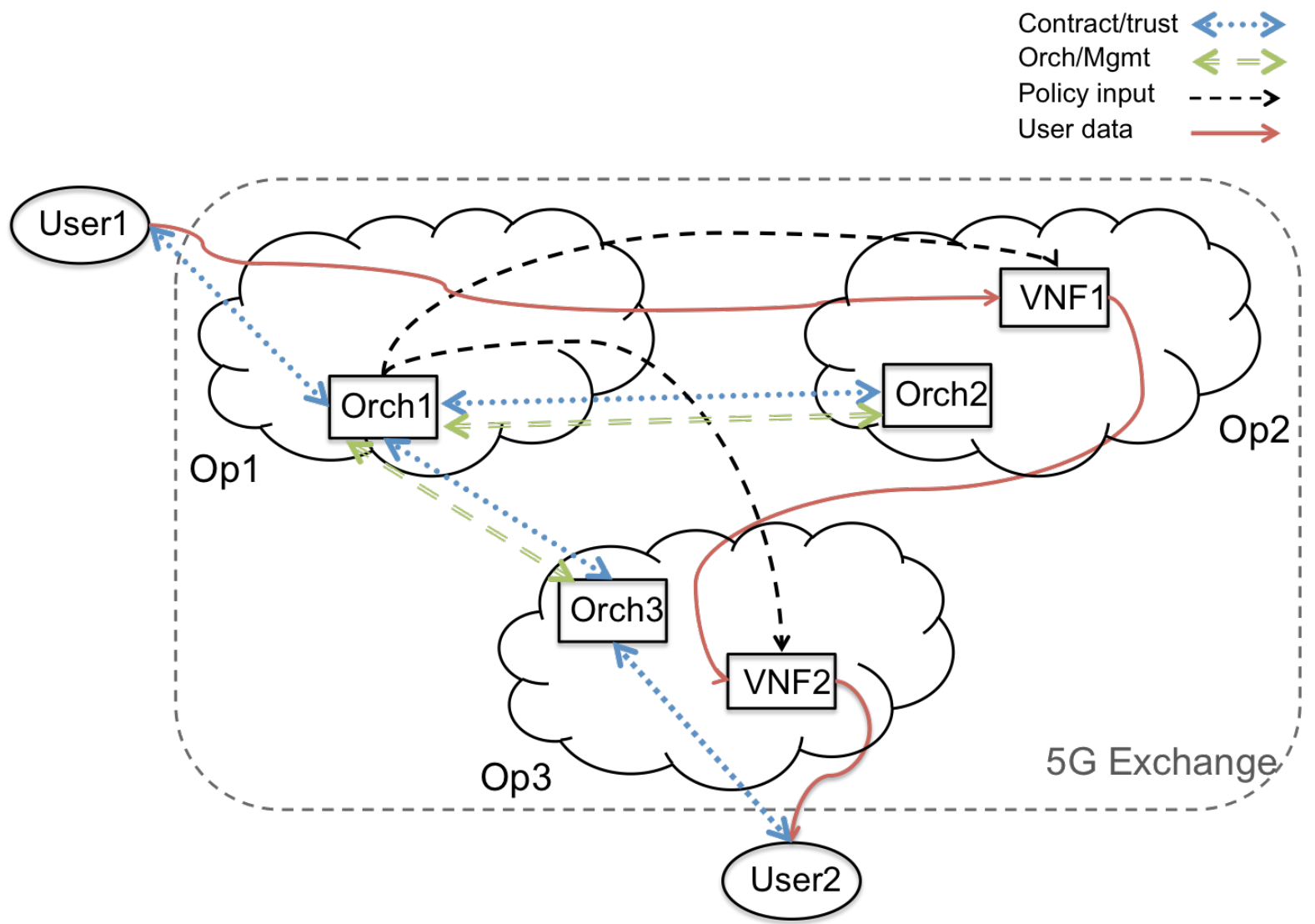

Figure $4 \quad$ Example: information flows in a 5GEx scenario

In order to rise up to this challenge, the following research roadmap could be followed. First, we have to understand what is the maximum amount and finest granularity of information we could possibly collect using advanced SDN and cloud monitoring techniques. Second, we should carefully investigate and quantify the interdependence of virtual compute, storage and network resources with regard to both performance and dependability, both within a domain and over multiple domains, or if they share some physical resources. Third, we should repeat step 2 upwards in the value chain to have a model of the whole ecosystem. Finally, we should go beyond basic aggregated mean values when it comes to KPIs, and consider quantiles and even full probability distributions of important performance and dependability metrics, potentially leading to more descriptive SLAs, supporting assured quality service delivery.

\section{Business and operational impact}

In summary, driven by the $5 \mathrm{G}$ technology innovations and enablement of new verticals and their diversity of future applications, there are numerous economical drivers to push and reshape the overall response by the industry in terms of multi-provider services. We foresee a future where business and technology enablers will be carefully aligned and provide an agile, efficient and open multi-service multi-provider solution. Enabled by SDN we anticipate an evolution into a powerful multi-service platform that goes beyond pure connectivity offerings. 
This multi-provider platform will also accommodate the needs of, as well as integrate the capabilities of, the emerging NFV and softwarization solutions.

While learning from the IT and cloud industry, the Telco and NSP industry will find themselves in an even more uncertain position and challenged with many strategic questions. On the one hand, the new technology enablers will allow on-demand trading, flexibility in re-negotiating SLAs, elasticity and dynamic traffic, resource and service management. However, which resources should you own and which resources and services should you buy? What is the best contract duration, and the better roadmap for my service and capability offerings? What kind of partnerships should I develop? How will I best adjust my organization and my operational processes to become an excellent player in such a future [14]? Perhaps the biggest challenge to the network infrastructure and services is "bootstrapping" the basic solution enablers when faced with so many multi-stakeholder coordination issues. On the positive side, solution proposals are now getting more mature and business attention is rising as the need for solving the challenges are becoming more clear. The use cases and $5 \mathrm{G}$ verticals mentioned above are good examples for that.

SDN-enabled solutions can help NSPs evolve their networking solutions in manageable steps according to their business developments and roadmap. By evolving the current IP traffic exchange solutions, it can complement and augment the current BGP-based operations with managed quality and multi-service inter-NSP 5G-ready traffic exchange services and SLA management solutions. When such a managed and assured quality traffic exchange solution is enabled among a set of initial partners, the solution can scale and grow into a full fledge traffic, resource and service trading and exchange platform. This holistic multi-domain resource slicing solution will unleash the full potential of $5 \mathrm{G}$.

The value-added connectivity and services that are specific to the end-customer are handled by appropriate policies at the SDN-enabled service edge nodes. The traffic flows are then steered onto the appropriate infrastructure ASQ paths and back-office data centers, according to the application requirements. This way, consistent end-to-end traffic and service handling across domains can be achieved and supported by the intelligence of SDN controllers and the SDN-enabled monitoring and service assurance capabilities. The above anticipated multidomain service and resource orchestration, hierarchical SLA management, and the need for automated mapping between high-level and lower level SLAs and their specific configurations and monitoring capabilities will become more crucial as the industry evolves [15].

\section{Conclusions}

The advent of the $5 \mathrm{G}$ era brings with itself the promise of value creation by means of a wide range of verticals. On the one hand, we have demonstrated how today's best effort Internet is not suitable for the assured quality interconnection these inherently multi-operator services require; other existing solutions are either too domain-specific or have incomplete functionality. On the other hand, we have shown which features of SDN and NFV technologies supplemented with service function chaining serve as key enablers for a novel alternative concept called 5G Exchange (5GEx). We have introduced the 5GEx conceptual architecture and presented how it is able to handle the inter-operator orchestration of composite (network and cloud) resources with the main technical concept of resource slicing. We have also 
outlined the 5GEx value proposition enabling the creation and trading of complex, high margin services built on top of low margin, commodity building blocks. Furthermore, we have addressed the open question of how to exchange information within the 5GEx framework and provided a roadmap for future research. Finally, we have investigated the business and operational impact of the envisioned solution. We believe that the $5 \mathrm{G}$ Exchange is capable of satisfying both the technical and business requirements of future $5 \mathrm{G}$ verticals and ushering us into the $5 \mathrm{G}$ era.

\section{Acknowledgments}

This work has been performed in the framework of the H2020-ICT-2014 project 5GEx (Grant Agreement no. 671636), which is partially funded by the European Commission. G. Biczók and L. Toka have been supported by the János Bolyai Research Scholarship of the Hungarian Academy of Sciences.

\section{References}

[1] NGMN Alliance, "5G White Paper," Feb. 2015 [Online.] Available: https:// www.ngmn.org/uploads/media/NGMN 5G White Paper V1 0.pdf. Accessed on October 27th, 2016.

[2] D. Weller and B. Woodcock, "Internet Traffic Exchange: Market Developments and Policy Challenges". OECD Digital Economy Papers No. 207, 2012

[3] J. Walrand, "Economic Models of Communication Networks", in Performance Modeling and Engineering, in Z. Liu et al., Eds., Springer, 2008

[4] P. Buccirossi et al., "Competition in the Internet Backbone Market", World Competition, vol. 28, no. 2, April 2005, pp. 235-254

[5] EU FP7 Project ETICS [Online.] Available: http://www.ict-etics.eu. Accessed on October 27th, 2016.

[6] GSM Association, "Guidelines for IPX Provider networks", version 9.1, May 2013

[7] OnApp [Online.] Available: http://onapp.com. Accessed on October 27th, 2016.

[8] CloudStore [Online.] Available: http://govstore.service.gov.uk/cloudstore/. Accessed on October 27th, 2016.

[9] A. Gupta et al., "SDX: A Software Defined Internet Exchange", in Proc. 2014 ACM SIGCOMM, Chicago, IL, 2014, pp. 551-562

[10] EU H2020 5GPPP Project 5G Exchange [Online.] Available: http://www.5gex.eu. Accessed on October 27th, 2016.

[11] EU FP7 Project UNIFY [Online.] Available: http://www.fp7-unify.eu. Accessed on October 27th, 2016.

[12] EU FP7 Project T-NOVA [Online.] Available: http://www.t-nova.eu. Accessed on October 27th, 2016.

[13] Ericsson, "5G Systems: Enabling Industry and Society Transformation", Ericsson White Paper, January 2015

[14] L. M. Contreras et al., "Operational, organizational and business challenges for network operators in the context of SDN and NFV", Computer Networks, vol. 92, no. 2, pp. 211-217, December 2015

[15] P. Wieder et al., Eds., "Service Level Agreements for Cloud Computing", Springer Science \& Business Media, 2011 


\section{Biographies}

GERGELY BICZÓK [S'03, M'11] is a researcher at the MTE-BME Future Internet Research Group and an assistant professor at the Budapest Univ. of Technology of Economics, where he received his $\mathrm{PhD}$ in Computer Science in 2010. Previously, he was a postdoc at the Norwegian University of Science and Technology, a Fulbright scholar at Northwestern University and a research fellow at Ericsson Research. His research interests center around the economics of networked systems.

MANOS (short for EMMANOUIL) DRAMITINOS is researcher in the Department of Informatics of AUEB. His research interests include network economics and traffic management, mobile networks, auction theory, business modeling and regulation. He has published high-quality papers in scientific journals and conferences and is the author of the book "Auction Theory for Telecoms".

LASZLO TOKA is currently a researcher with the MTA-BME Information Systems Research Group and an assistant professor at the Budapest University of Technology and Economics. He received his PhD in 2011 at Telecom Paris, and afterwards worked at Ericsson Research for 3 years before rejoining academia. His main research topics lie within software-defined networking, data analytics, and economic modeling of distributed systems.

POUL HEEGAARD [SM'14] received his Ph.D. in telematics from NTNU in 1998. He has been a full professor at NTNU since 2010. His main research interests are performance and dependability modelling and simulations of communication networks, currently focusing on resource optimization and management in distributed autonomous systems in a multi-domain context. He was head of the Department of Telematics (2009-2013), and is now head of the NTNU QUAM (Quantitative modelling of dependability and performance) research lab.

HÅKON LØNSETHAGEN is a Senior Adviser at Telenor Research. He received his B.Sc. in EE and CS from the University of Colorado, Boulder in 1987 and an M. Sc. from the Norwegian Institute of Technology in 1988. His current focus is on inter-NSP differentiated and assured services quality and business models, and SDN and NFV. He has participated and been WP and task leader in Eurescom projects and EU IST DAVID, NOBEL, NOBEL2, ETICS and 5GEx. He has made several contributions to TM Forum and been an advisory board member of EU research projects SmartenIT and NEAT. 